\title{
Better operative outcomes achieved with the prone jackknife vs. lithotomy position during abdominoperineal resection in patients with low rectal cancer
}

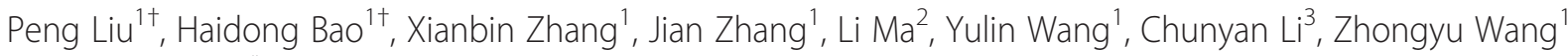 \\ and Peng Gong ${ }^{1 *}$
}

\begin{abstract}
Background: Lithotomy (LT) and prone jackknife positions (PJ) are routinely used for abdominoperineal resection (APR). The present study compared the clinical, pathological, and oncological outcomes of PJ-APR vs. LT-APR in low rectal cancer patients in order to confirm which position will provide more benefits to patients undergoing APR.

Methods: This is a retrospective study of consecutive patients with low rectal cancer who underwent curative APR between January 2002 and December 2011. Patients were matched 1:2 (PJ-APR $=74$ and LT-APR $=37$ patients) based on gender and age. Perioperative data, postoperative outcomes, and survival were compared between the two approaches.

Results: Hospital stay was shorter with PJ-APR compared with LT-APR $(P<0.05)$. Compared with LT-APR, duration of anesthesia ( $234 \pm 50.8$ vs. $291 \pm 69 \mathrm{~min}, P=0.022)$ and surgery (183 \pm 44.8 vs. $234 \pm 60 \mathrm{~min}, P=0.016$ ) was shorter with PJ-APR, and estimated blood losses were smaller ( $549 \pm 218$ vs. $674 \pm 350 \mathrm{~mL}, P<0.001)$. Blood transfusions were required in $37.8 \%$ of LT-APR patients and in $8.1 \%$ of PJ-APR patients $(P<0.001)$. There was no difference in the distribution of $\mathrm{N}$ stages $(P=0.27)$. Median follow-up was $47.1(13.6-129.7)$ months. Postoperative complications were reported by fewer patients after PJ-APR compared with LT-APR (14.9\% vs. 32.4\%, $P=0.030)$. There were no significant differences in overall survival, disease-free survival, local recurrence, and distant metastasis $(P>0.05)$.
\end{abstract}

Conclusions: The PJ position provided a better exposure for low rectal cancer and had a lower operative risk and complication rates than LT-APR. However, there was no difference in rectal cancer prognosis between the two approaches. PJ-APR might be a better choice for patients with low rectal cancer.

Keywords: Rectal cancer, Abdominoperineal resection, Lithotomy position, Prone jackknife position

\section{Background}

Abdominoperineal resection (APR) remained for many years the main treatment option for most patients with low rectal cancer, despite high local recurrence and poor prognosis [1-5]. APR was gradually replaced by anterior resection (AR) and low anterior resection (LAR) for tumors of the upper and middle rectum [6]. However,

\footnotetext{
*Correspondence: medscigp@126.com

${ }^{\dagger}$ Equal contributors

${ }^{1}$ Department of General Surgery, The First Affiliated Hospital of Dalian

Medical University, 222 Zhongshan Road, 116011 Dalian, China

Full list of author information is available at the end of the article
}

APR is still required in selected cases of low rectal cancer [7-9].

Lithotomy (LT) and prone jackknife (PJ) positions are the two main positions currently used in APR. The LT position provides adequate access to the rectovaginal septum and allows easy access to the posterior face of the rectum [10]. However, this position is uncomfortable for the surgeons and the assistants, blood tends to accumulate in the operation area, and adequate lighting is often challenging. The PJ position is appropriate for almost all proctological surgeries. It allows an excellent exposure of the posterior and anal perineum and the anterior face of the rectum,

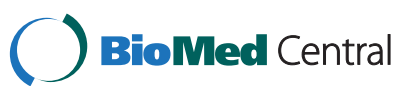


provides a more comfortable position for the surgeon and assistants, results in less blood accumulation, and enables better lighting. Specimens are also more easily obtained, with less circumferential resection margin (CRM) involvement than with the LT position $[11,12]$. A previous study has suggested that the prone position results in shorter operative time and in lower incidence of perineal wound infection, but the researchers did not look at the oncological outcomes [13]. Another study has suggested that there might be no difference in perioperative morbidity and oncological outcomes between the PJ and LT positions [14].

Therefore, the oncological outcomes are still in need of a better assessment. The objective of the present study was to compare these two approaches in terms of operative and oncological outcomes, in order to assess the impact of the position on the operative and oncological outcomes of patients undergoing APR.

\section{Methods}

\section{Study population}

In this retrospective study, we included all consecutive patients who underwent radical APR for stages I to III low rectal cancers at the First Affiliated Hospital of Dalian Medical University between January 2002 and December 2011. Inclusion criteria were 1) low rectal cancer, 2) absence of distant metastases, and 3) APR aiming to curative resection (R0).

Exclusion criteria were 1) inflammatory bowel diseases, 2) hereditary colorectal cancer syndromes, 3) recurrent cancer after anus-preserving surgery, 4) non-rectal cancer history, or 5) lost to follow-up or incomplete follow-up data. No patient received neoadjuvant therapy.

Pre-surgical assessment and staging were made using physical and laboratory examinations, including nutritional status, digital rectal examination, proctoscopy, tumor distance from the dentate line, colonoscopy, serum carcinoembryonic antigen (CEA) levels, chest radiography, chest computed tomography (CT) scan, abdominal and pelvic CT scan, endorectal ultrasound, and/or pelvic magnetic resonance imaging (MRI).

The present study was approved by the ethics committee of the First Affiliated Hospital of Dalian Medical University (\# LCKY2012-34), and the need for individual consent was waived.

Because the number of patients was higher in the PJAPR group $(n=192)$ than in the LT-APR group $(n=37)$, 1:2 matching of eligible patients was performed based on gender and age. Matching was done to decrease the possible bias due to the difference in the number of patients between the groups.

\section{Data collection}

Data were collected from the electronic medical records, including demographic data, clinical and pathological parameters, tumor-node-metastasis (TNM) status, adjuvant chemotherapy and/or radiation therapy, operative parameters, perioperative morbidity and mortality, postoperative outcomes, and oncological outcomes (overall survival, cancer-specific mortality, distant metastases, and local recurrence). TNM staging was based on the seventh edition of the AJCC TNM staging manual.

\section{Surgical techniques}

The operative position was selected by the surgeon according to his preoperative evaluation. All operations were performed by skilled colorectal surgeons with emphasis on the oncological principles of TME.

In PJ-APR, anal closure with purse string sutures was followed by an elliptical cutaneous incision 2 to $3 \mathrm{~cm}$ from the anal margin. The subcutaneous fat was divided and ligated laterally to expose both sides of the medial margin of the gluteus maximus muscle. Adipose tissue in the ischiorectal fossa was removed following anal vein ligation. The pelvic floor was identified and opened just anterior to the coccyx. The levator ani were then divided laterally as near as possible to its bony insertion to the pelvic sidewalls beginning from the posterior midline, and the dissection line joined the previously developed presacral plane of dissection.

The perineal wound was closed with a pelvic drain, opening near the incision. Patients were then turned into the supine position. All patients underwent high ligation of the inferior mesenteric artery and complete excision of the mesorectum. Sharp dissection of the mesorectum was performed in the avascular plane between the visceral fascia and the presacral fascia [15]. Care was taken to preserve the preaortic sympathetic plexus and hypogastric nerves. Laterally, the pelvic plexus was also preserved unless there was evidence of tumor invasion; the sacral nerve roots were preserved [8]. A permanent sigmoid colon colostomy was performed.

The first step of LT-APR was performed in the supine position. After establishment of a permanent sigmoid colon colostomy, patients were turned into the LT position, and the perineal dissection was then performed. The dissection was performed along similar planes and with the same goals as in PJ-APR, i.e., dissecting the levator ani entirely from the pelvic sidewalls [14], working circumferentially from posterior to anterior, and resulting in en bloc resection of the tumor and the adjacent pelvic floor without opening the interface between the tumor and the levator ani. Posterior vaginal resection was performed en bloc with the specimen if a tumor involving the anterior rectal wall was adherent to the vagina. Occasionally, partial prostatectomy was required [8].

\section{Surgical aims}

Curative intent was defined as the complete removal of gross tumor and tumor-associated lymph nodes, confirmed 
by pathological examination. Photographic documentation and the pathological examination of the specimen were performed by at least two pathologists with a special interest in colorectal surgery.

Blood loss was estimated based on the amount of blood in the aspiration devices minus the amount of saline used, and on the amount of blood in the gauzes, estimated using a gravimetric method [16].

\section{Postoperative follow-up}

Patients were followed up at 3-month intervals during the first 2 years, then every 6 months until 5 years. The follow-up was performed by the colorectal surgeons. Routine postoperative examination included a physical examination, blood CEA levels, colonoscopic examinations, chest X-rays, abdominopelvic CT or MRI scan, and/or wholebody bone scan.

Wound infection was defined as an infection of the surgical incision. The infection had to occur within 30 days of surgery, and the culture had to be positive.

Local recurrence within the pelvis was proven by 1) positive histology, 2) diagnostic imaging evidence with raised CEA levels, or 3) macroscopic evidence of tumor recurrence at laparotomy [17]. The presence of a tumor at any other site was defined as distant metastasis.

Disease-free survival was the time from the date of the primary treatment to the date of the first instance of recurrent disease (either local, systemic, or both if they occurred less than 6 months apart). Overall survival was defined from the date of the primary treatment to the date of death from any cause [18].

For the purpose of the study, follow-up was closed on March 31, 2013.

\section{Statistical analysis}

Statistical analysis was performed using SPSS 16 (SPSS Inc., Chicago, IL, USA). Categorical variables were compared using chi-square or Fisher's exact tests, as appropriate. Continuous variables are presented as means \pm SD and were compared using independent samples $t$-tests. Kaplan-Meier curves and log-rank tests were used to evaluate oncological outcomes. Multivariate Cox regression analysis was performed for survival outcome. The dependent variable was survival, and the independent variables included gender, age, surgical position, tumor size, pathological type, tumor differentiation, pathologic $\mathrm{T}$ and $\mathrm{N}$ stages, vascular tumor embolus, postoperative radiotherapy, and chemotherapy. $P<0.05$ was considered statistically significant.

\section{Results}

\section{Patients' characteristics}

Between January 2002 and December 2011, 383 patients underwent curative APR for low rectal cancer; 154 patients were excluded from the analysis, and 229 patients with primary rectal cancer were included. Among them, 192 (83.8\%) underwent PJ-APR and 37 (16.2\%) underwent LT-APR; 147 were men, and 82 were women. Median age was 65 years (range: 28 to 86 ).

After matching, there were 37 patients in the LT-APR group and 74 patients in the PJ-APR group. Besides the matching criteria, there were no differences in PTNM stage, postoperative chemo- and radiotherapy, histological grade, pathological type, body mass index, tumor stage, and duration of liquid and semi-liquid diets between the two groups (all $P>0.05$ ) (Table 1$)$.

\section{Perioperative and short-term postoperative outcomes}

Anesthesia duration and operative time were both significantly shorter in patients who underwent PJ-APR (anesthesia: $234 \pm 51$ vs. $291 \pm 69 \mathrm{~min}, P=0.002$; operation: $183 \pm 45$ vs. $234 \pm 60 \mathrm{~min}, P=0.016$ ) (Table 1 ). In addition, estimated blood loss during PJ-APR was significantly less than during LT-APR $(549 \pm 218$ vs. $674 \pm$ $350 \mathrm{~mL}, P<0.001)$, and more patients who underwent LT-APR required blood transfusion $(P<0.001)$ (Table 1$)$. There were no significant differences in the distance of the tumor to the dentate line, tumor size, pathological type, differentiation, and $\mathrm{T}$ or $\mathrm{N}$ stage (Table 1). The specimens were assessed by at least two pathologists. Results of the pathological examination showed that the surgical margins of the specimens were negative.

Two patients in the PJ-APR group and two in the LTAPR group experienced complications during the operation $(P=0.407)$ (Table 2). No female patient suffered from vaginal injury and hemorrhage during operation. After surgery, 11 (14.9\%) of the 74 patients who underwent PJ-APR experienced postoperative complications, compared with $12(32.4 \%)$ of the 37 patients who underwent LT-APR $(P=$ 0.030; Table 2). Occurrence of perineal wound infection (1.4\%) and incision fat necrosis (1.4\%) was significantly lower in the PJ-APR group than in the LT-APR $(10.8 \%$ and $16.2 \%$, respectively, both $P<0.05$ ). There was no difference in the rate of abdominal infection $(4.1 \%$ vs. $8.1 \%, P=0.317)$ (Table 2).

\section{Long-term oncological outcomes}

By March 2013, 64 (57.7\%) patients were alive at a median follow-up of 47.1 (13.6 to 129.7) months. A total of 47 patients (42.3\%) died during the study period. The causes of death included local recurrence $(n=14)$, distant metastases $(n=22)$, or both $(n=8)$. Two patients died from heart disease, and the last one died in a car accident.

There were 44 (59.5\%) patients alive in the PJ-APR group after a median follow-up of 38.5 (7.8 to 122.4) months, and 20 (54.1\%) patients in the LT-APR group were alive after a median follow-up of 47.4 (2.6 to 129.7$)$ months $(P=0.593)$ (Table 3 and Figure 1A). 
Table 1 Perioperative variables according to patient positioning

\begin{tabular}{|c|c|c|c|c|}
\hline Variables & & PJ-APR $(n=74)$ & LT-APR $(n=37)$ & $P$ value \\
\hline \multirow[t]{2}{*}{ Gender, $n(\%)$} & Male & $52(70)$ & $26(70)$ & \multirow[t]{2}{*}{0.591} \\
\hline & Female & $22(30)$ & $11(30)$ & \\
\hline Age, years & & $63.0 \pm 10.5(39-83)$ & $64.0 \pm 11.7(39-84)$ & 0.396 \\
\hline Body mass index, $\mathrm{kg} / \mathrm{m}^{2}$, mean (range) & & $23.2 \pm 3.3(16.4-29.4)$ & $22.9 \pm 3.2(16.8-31.2)$ & 0.581 \\
\hline Length of hospital stay, days & & $23 \pm 7.4(11-46)$ & $29 \pm 11.7(17-63)$ & 0.011 \\
\hline Duration of anesthesia, min & & $234 \pm 51(130-385)$ & $291 \pm 69(150-450)$ & 0.022 \\
\hline Operative time, min & & $183 \pm 45(90-320)$ & $234 \pm 60(120-370)$ & 0.016 \\
\hline \multirow[t]{2}{*}{ Operative time, $n(\%)$} & $\leq 120$ & $6(8.1)$ & $1(2.7)$ & \multirow[t]{2}{*}{0.255} \\
\hline & $>120$ & $68(91.9)$ & $36(97.3)$ & \\
\hline Estimated blood loss, $\mathrm{mL}$ & & $549 \pm 218(200-1,137)$ & $674 \pm 350(160-1,440)$ & $<0.001$ \\
\hline Blood transfusion, $n$ (\%) & & $6(8.1)$ & $14(37.8)$ & $<0.001$ \\
\hline Tumor distance, $\mathrm{cm}$ & & $4.0 \pm 1.4(1-10)$ & $3.9 \pm 1.1(2-7)$ & 0.282 \\
\hline Tumor size, $\mathrm{cm}$ & & $4.4 \pm 1.8(1.5-12)$ & $4.7 \pm 1.3(2.4-8)$ & 0.221 \\
\hline \multirow[t]{3}{*}{ Macroscopic type of tumor, $n(\%)$} & Mushroom & $19(25.7)$ & $6(16.2)$ & \multirow[t]{3}{*}{0.486} \\
\hline & Ulcerative & $54(72.9)$ & $30(81.1)$ & \\
\hline & Infiltrative & $1(1.4)$ & $1(2.7)$ & \\
\hline \multirow[t]{3}{*}{ Pathological type, $n(\%)$} & Adenocarcinoma & 73 (98.6) & $36(97.3)$ & \multirow[t]{3}{*}{0.286} \\
\hline & Squamous carcinoma & $1(1.4)$ & 0 & \\
\hline & Rectal carcinoid & 0 & $1(2.7)$ & \\
\hline \multirow[t]{3}{*}{ Degree of differentiation, $n(\%)$} & Good & $4(5.4)$ & $1(2.7)$ & \multirow[t]{3}{*}{0.537} \\
\hline & Moderate & $49(66.2)$ & $28(75.7)$ & \\
\hline & Poor & $21(28.4)$ & $8(21.6)$ & \\
\hline \multirow[t]{4}{*}{ Pathologic T stage, $n$ (\%) } & $\mathrm{T} 1$ & 0 & $1(2.6)$ & \multirow[t]{4}{*}{0.468} \\
\hline & $\mathrm{T} 2$ & $16(21.6)$ & $10(27)$ & \\
\hline & T3 & $54(73.0)$ & $24(65)$ & \\
\hline & T4 & $4(5.4)$ & $2(5.4)$ & \\
\hline \multirow[t]{3}{*}{ Pathologic N stage, $n(\%)$} & No & $36(48.6)$ & $20(54.1)$ & \multirow[t]{3}{*}{0.269} \\
\hline & N1 & $26(35.1)$ & $15(40.5)$ & \\
\hline & N2 & $12(16.2)$ & $2(5.4)$ & \\
\hline Time of fluid diet, days & & $4.2 \pm 1.2(2-8)$ & $4.0 \pm 0.9(2-7)$ & 0.055 \\
\hline Time of semi-liquid diet, days & & $7.1 \pm 1.9(4-14)$ & $6.7 \pm 3.3(3-22)$ & 0.386 \\
\hline Pelvic drain time, days & & $10.5 \pm 3.1(5-22)$ & $9.4 \pm 3.8(5-20)$ & 0.338 \\
\hline Postoperative hospitalization, days & & $15.6 \pm 5.3(7-35)$ & $22 \pm 10.8(10-51)$ & $<0.001$ \\
\hline \multirow[t]{2}{*}{ Postoperative chemoradiotherapy, $n$ (\%) } & Yes & $35(47.3)$ & $15(40.5)$ & \multirow[t]{2}{*}{0.319} \\
\hline & No & $39(52.7)$ & $22(59.5)$ & \\
\hline
\end{tabular}

$A P R$ abdominoperineal resection, $P J-A P R$ abdominoperineal resection in prone jackknife position, $L T$-APR abdominoperineal resection in lithotomy position.

The overall disease-free survival was $52.7 \%$ (39/74) in the PJ-APR group and 51.4\% (19/37) in the LT-APR group $(P=0.325)$ (Table 3 and Figure 1B). The rates of local recurrence and distant metastasis were $17.6 \%(13 / 74)$ and $23 \%(17 / 74)$ in the PJ-APR group, and $18.9 \%(7 / 37)$ and $24.3 \%(9 / 37)$ in the LT-APR group $(P>0.05$, Table 3$)$.

Between January 2002 and February 2008, 60 patients who underwent APR had a follow-up of more than 5 years. Among this subgroup, the overall 5-year survival was
$46.7 \%(28 / 60)$, and the overall 5 -year disease-free survival was $45 \%(27 / 60)$. There was no significant difference in the overall 5-year survival between the PJ-APR (50\%, $16 / 32)$ and LT-APR groups $(42.9 \%, 12 / 28 ; P=0.821)$ (Table 3, Figure 1C), as well as for the 5 -year diseasefree survival rate $(46.9 \%$ vs. $42.9 \% ; P=0.638)$ (Table 3 , Figure 1D).

The rates of local recurrence (9.4\%) and distant metastasis $(21.9 \%)$ in the PJ-APR group were not significantly 
Table 2 Complications of APR according to patient positioning

\begin{tabular}{|c|c|c|c|}
\hline Variables & PJ-APR, $(n=74)$ & LT-APR, $(n=37)$ & $P$ value \\
\hline \multicolumn{4}{|l|}{ Intraoperative complications, $n(\%)$} \\
\hline Total & $2(2.7)$ & $2(5.4)$ & 0.407 \\
\hline latrogenic tumor perforation & $2(2.7)$ & $2(5.4)$ & 0.407 \\
\hline Vaginal injury & 0 & 0 & NA \\
\hline Urethral/ureteric injury & $1(1.4)$ & $1(2.7)$ & 0.558 \\
\hline Hemorrhage & 0 & 0 & NA \\
\hline \multicolumn{4}{|l|}{ Postoperative complications, n (\%) } \\
\hline Total & $11(14.9)$ & $12(32.4)$ & 0.030 \\
\hline Urinary retention & $7(9.5)$ & $7(18.9)$ & 0.134 \\
\hline Abdominal wound infection & $3(4.1)$ & $3(8.1)$ & 0.317 \\
\hline Perineal wound infection & $1(1.4)$ & $4(10.8)$ & 0.042 \\
\hline Incision fat necrosis & $1(1.4)$ & $6(16.2)$ & 0.005 \\
\hline Incision dehiscence & $1(1.4)$ & $3(8.1)$ & 0.107 \\
\hline Intestinal obstruction & 0 & $1(2.7)$ & 0.333 \\
\hline DVT (deep venous thrombosis) & $1(1.4)$ & $1(2.7)$ & 0.558 \\
\hline Pelvic abscess & $1(1.4)$ & $1(2.7)$ & 0.558 \\
\hline Reoperation & $1(1.4)$ & 0 & 0.667 \\
\hline Readmission & $3(4.1)$ & $2(5.4)$ & 0.542 \\
\hline
\end{tabular}

$A P R$ abdominoperineal resection, $P J-A P R$ abdominoperineal resection in prone jackknife position, $L T$-APR abdominoperineal resection in lithotomy position, NA not applicable, DVT deep venous thrombosis.

different from those in the LT-APR group $(21.4 \%$ and 28.6\%; all $P>0.05$ ) (Table 3).

Fifty-two patients received postoperative radiation therapy and/or chemotherapy. The Kaplan-Meier analysis indicated that postoperative therapy did not affect the overall survival rate in all patients $(P=0.643$, Figure $2 \mathrm{~A})$, or in the subgroup with a follow-up of at least 5 years $(P=0.500$, Figure $2 \mathrm{~B})$.

Cox regression analysis identified gender and pathologic $\mathrm{N}$ stage as independent prognostic factors, but operative position of the patient was not a significant factor in this analysis (Table 4).

Table 3 Oncological outcomes according to patient positioning

\begin{tabular}{llll}
\hline Oncological outcomes & PJ-APR & LT-APR & P value \\
\hline Survival & $59.5 \%(44 / 74)$ & $54.1 \%(20 / 37)$ & 0.593 \\
Disease-free survival & $52.7 \%(39 / 74)$ & $51.4 \%(19 / 37)$ & 0.325 \\
Local recurrence & $17.6 \%(13 / 74)$ & $18.9 \%(7 / 37)$ & 0.995 \\
Distant metastasis & 23.0\%(17/74) & $24.3 \%(9 / 37)$ & 0.811 \\
5-year survival & $50.0 \%(16 / 32)$ & $42.9 \%(12 / 28)$ & 0.821 \\
5-year disease-free survival & $46.9 \%(15 / 32)$ & $42.9 \%(12 / 28)$ & 0.638 \\
5-year local recurrence & $9.4 \%(3 / 32)$ & $21.4 \%(6 / 28)$ & 0.173 \\
5-year distant metastasis & $21.9 \%(7 / 32)$ & $28.6 \%(8 / 28)$ & 0.382 \\
\hline
\end{tabular}

PJ-APR abdominoperineal resection in prone jackknife position, $L T-A P R$ abdominoperineal resection in lithotomy position.

\section{Discussion}

APR is a complex and challenging surgical procedure. The present study compared the clinical, pathological, and oncological outcomes of PJ-APR vs. LT-APR in low rectal cancer patients in order to confirm which position will provide more benefits to patients undergoing APR. The present study showed that the PJ position was superior to the LT position in terms of operative outcomes. Indeed, compared with LT-APR, the duration of anesthesia and surgery was shorter by $>50 \mathrm{~min}$, estimated blood loss in PJ-APR was reduced by $>100 \mathrm{~mL}$, and fewer patients needed blood transfusions with PJ-APR. Furthermore, postoperative hospitalization was significantly shorter with PJ-APR, and some of the postoperative complications were significantly reduced with PJ-APR. There was no difference in oncological outcomes between PJ-APR and LT-APR including overall survival, disease-free survival, local recurrence, and distant metastases. These results are supported by previous studies [14].

Perineal dissection is complicated in APR, and performing APR in the PJ position improved visualization, reduced the risks related to the operation, and made the operation easier to perform during this difficult part. Although the LT position provides adequate access to the rectovaginal septum and allows easy access to the posterior face of the rectum [10], this position is uncomfortable for the surgeons and assistants, blood tends to accumulate in the operation area, and adequate lighting 

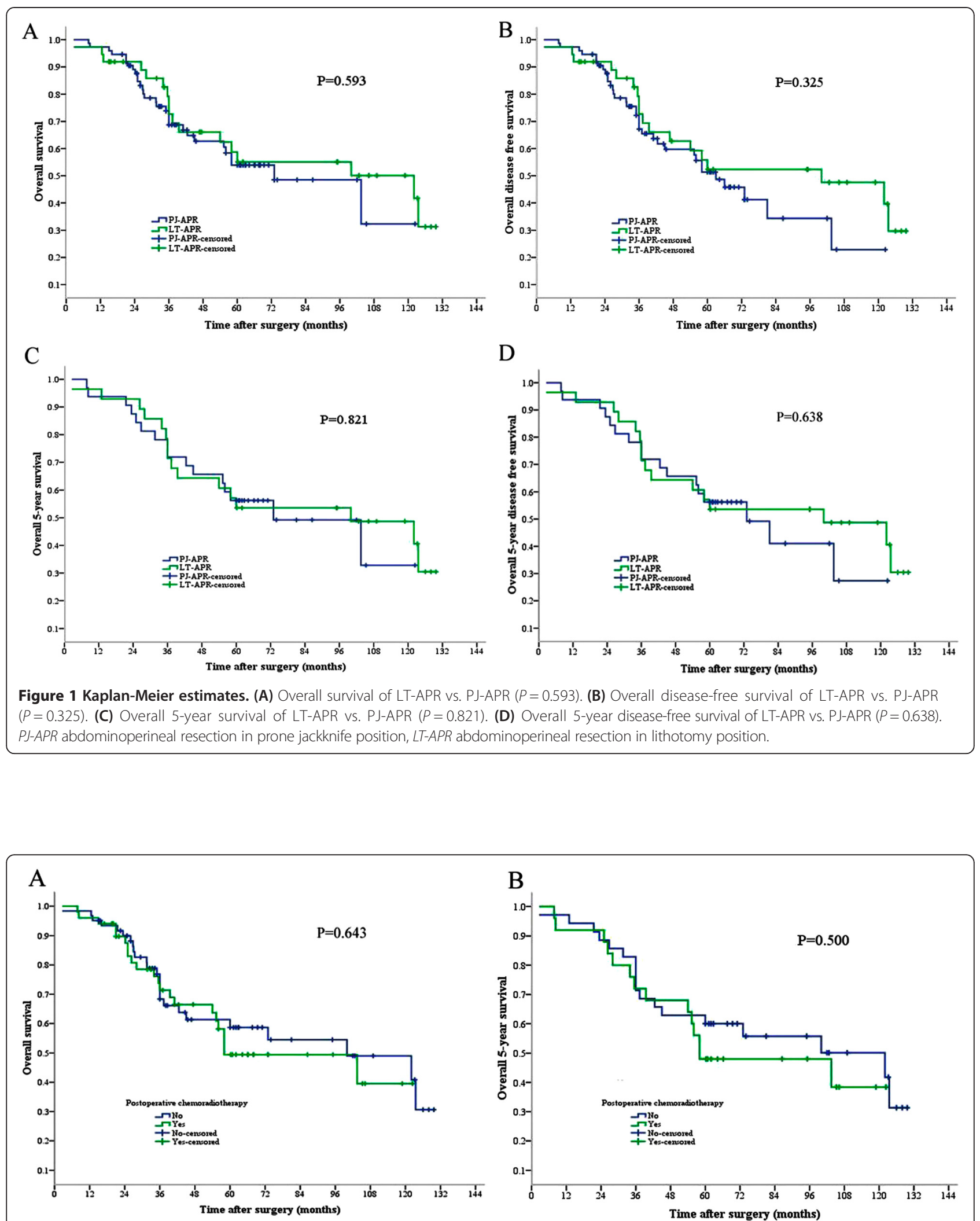

Figure 2 Chemoradiotherapy and oncological outcomes. (A) Postoperative chemoradiotherapy and oncological outcomes $(P=0.643)$. (B) Postoperative chemoradiotherapy and 5 -year oncological outcomes $(P=0.500)$. PJ-APR abdominoperineal resection in prone jackknife position, LT-APR abdominoperineal resection in lithotomy position. 
Table 4 Cox regression analysis of factors associated with survival

\begin{tabular}{lllll}
\hline \multicolumn{2}{l}{ Clinicopathologic factors } & HR & $\mathbf{9 5 \% ~ C l}$ & $\boldsymbol{P}$ value \\
\hline Gender & Male & 1 & & \\
& Female & 0.532 & $0.385-0.976$ & 0.035 \\
Pathologic N stage & No & 1 & & \\
& N1 & 4.147 & $2.377-5.890$ & 0.021 \\
& N2 & 6.738 & $2.592-18.661$ & 0.002 \\
\hline
\end{tabular}

is often challenging. Therefore, the surgeon has to spend more time for exposure, operative vision, and hemostasis and also to avoid iatrogenic damage to nerves, blood vessels, and organs. Especially, the anterior wall of the rectum is more difficult to access. Although the patient position needs to be changed during the operation in PJAPR, this does not need a lot of time [14]. The PJ position is appropriate for almost all proctological surgeries, it allows an excellent exposure of the posterior and anal perineum and the anterior face of the rectum, provides a more comfortable position for the surgeon and assistants, results in less blood accumulation, and enables better lighting [10]. Our results showed that the time needed to change the operation position during PJ-APR was less than 18 min (data not shown); more time was saved in the process of perineal dissection. Moreover, data also showed that the estimated blood loss in PJ-APR was reduced by more than $120 \mathrm{~mL}$ compared with LT-APR and that the rate of patients needing blood transfusion was $6 \%$ during PJ-APR compared with $14 \%$ during LT-APR. In addition, postoperative hospitalization was significantly shorter after PJ-APR compared with LT-APR, which may indicate that patients recovered faster after PJ-APR than LT-APR.

APR requires a complex positioning and exposes the patients to the risks of a major surgery, as well as to the risks specific to this procedure [19]. Previous studies have shown that the incidence of intraoperative perforations was markedly reduced with PJ-APR compared with supine APR [12] and that intraoperative bowel perforation in extralevator APR was significantly reduced when the perineal dissection was performed in the PJ position rather than in the LT or Lloyd-Davies position [11]. The most common immediate complication is intra-abdominal or pelvic abscesses, which has been reported to account for $32 \%$ of postoperative complications [20]. The incidence of pelvic abscesses has been estimated at 3\% among patients who underwent APR, and it is generally recognized that a pelvic abscess requires reoperation or readmission more frequently than perineal wound infection [21]. Other studies have shown that the risk of postoperative sexual or urinary dysfunction ranges from $10 \%$ to $60 \%[14,22]$.

In the present study, the incidence of perineal wound infection and incision dehiscence was $1.4 \%$ in the PJ-APR group compared with $8 \%-16 \%$ in the LT-APR group. The incidence of pelvic abscesses was also lower with PJ-APR (1.4\%) than with LT-APR (2.7\%). These results are supported by a previous study (11\% vs. $36 \%$ ) [13], but a much lower incidence was observed in the present study. This low postoperative complication rate may be explained, at least in part, by the fact that all patients underwent curative APR, and not palliative surgery. The other reason is that patients with comorbidities were excluded. In addition, our patients did not receive any neoadjuvant therapy, which could partially explain the low rate of infection. Previous studies have reported infection rates as high as $50 \%$ and substantial morbidity, especially after neoadjuvant radiation and chemoradiation therapy [23,24].

Reported survival and local recurrence rates after APR for rectal cancer vary widely. APR is generally associated with poor oncological outcomes $[4,25,26]$. Indeed, the 5 -year cancer-specific survival after sphincter-sparing resection was $74 \%$ compared with $62 \%$ after APR [27]. However, the same group showed that 2 years after surgery, local recurrence rates were similar with both procedures [28].

A large database analysis suggested that there has been no improvement in mortality following APR from 1996 to 2004 [1]. In the present study, the perineal phase of APR was carried out in the PJ position, followed by abdominal surgery. Because the inferior mesenteric artery is not ligated during the same procedure phase, we were concerned that this may potentially facilitate arterial transport of cancer cells, resulting in metastasis and poor prognosis. However, results showed that overall survival, 5 -year overall survival, and oncological outcomes were comparable between the two groups. Cox regression analysis excluded the operative position as being an independent prognostic factor, while gender and pathologic $\mathrm{N}$ stage were independent factors associated with survival. Notably, there were no significant differences in pathologic $\mathrm{T}$ or $\mathrm{N}$ stages, or in the degree of differentiation. However, the number of patients with pT3 and/or poorly differentiated tumors was slightly higher in the PJ-APR group.

The present study suffers from some limitations. First, we were unable to account for the CRM, which has previously been associated with increased local recurrence and reduced patient survival [29-31]. Second, we included all histological subtypes of rectal cancer. However, adenocarcinoma largely predominates (98.3\%), and we do not think that the few cases of other subtypes could impact the results much. Third, because of the retrospective nature of the study, we are unable to obtain reliable data about the extralevator approach. Finally, each surgeon selected the operative position for each specific patient based on its appreciation of a number of factors, but there were no specific criteria for LT or PJ. In addition, for a specific 
patient, a surgeon may have selected the LT position, while another surgeon would have selected the PJ position. Therefore, we cannot provide any exact criteria. We agree that it could have introduced a bias, but results showed that the patients were comparable for most characteristics at baseline.

\section{Conclusions}

The present study suggests that the PJ position provides a better exposure for low rectal cancer and that the operative risks and complications were reduced by performing APR in the PJ position compared with the more conventional LT position. However, the oncological outcomes were not significantly improved. Therefore, PJAPR might be a better choice for patients with low rectal cancer to reduce operative risks and complications.

\section{Competing interests}

The authors declare that they have no competing interests.

\section{Authors' contributions}

$\mathrm{HB}$ and PL carried out the conception and design of the study, acquisition of data, and writing of the manuscript. LM carried out data analysis and interpretation of data. $X Z$ and $J Z$ participated in the acquisition of data. YW and CL carried out the acquisition of data, and analysis and interpretation of data. ZW carried out the acquisition of data. PG participated in the revision of the manuscript. All authors read and approved the final manuscript.

\section{Acknowledgements}

This study was funded by the Specialized Research Fund for the Doctoral Program of Higher Education (No. 20122105110009), the China National Natural Science Foundation (No. 81473504), and Research Award Fund for Outstanding Young Scientists (No. BS2011YY016). The funders had no role in study design, data collection and analysis, decision to publish, or preparation of the manuscript.

\section{Author details}

${ }^{1}$ Department of General Surgery, The First Affiliated Hospital of Dalian Medical University, 222 Zhongshan Road, 116011 Dalian, China. ${ }^{2}$ Department of Epidemiology, Dalian Medical University, 9 Lvshun Road South, 116044 Dalian, China. ${ }^{3}$ Department of Gastroenterology, The First Affiliated Hospital of Dalian Medical University, 222 Zhongshan Road, 116011 Dalian, China.

Received: 2 July 2014 Accepted: 8 January 2015

Published online: 12 February 2015

\section{References}

1. Tilney HS, Heriot AG, Purkayastha S, Antoniou A, Aylin P, Darzi AW, et al. A national perspective on the decline of abdominoperineal resection for rectal cancer. Ann Surg. 2008;247:77-84.

2. Engel AF, Oomen JL, Eijsbouts QA, Cuesta MA, van de Velde CJ. Nationwide decline in annual numbers of abdomino-perineal resections: effect of a successful national trial? Color Dis. 2003;5:180-4.

3. Martling AL, Holm T, Rutqvist LE, Moran BJ, Heald RJ, Cedemark B. Effect of a surgical training programme on outcome of rectal cancer in the County of Stockholm. Stockholm Colorectal Cancer Study Group. Basingstoke Bowel Cancer Research Project. Lancet. 2000;356:93-6.

4. Kim JS, Hur H, Kim NK, Kim YW, Cho SY, Kim JY, et al. Oncologic outcomes after radical surgery following preoperative chemoradiotherapy for locally advanced lower rectal cancer: abdominoperineal resection versus sphincter-preserving procedure. Ann Surg Oncol. 2009;16:1266-73.

5. Law WL, Chu KW. Abdominoperineal resection is associated with poor oncological outcome. Br J Surg. 2004;91:1493-9.
6. Collins DC. End-results of the Miles' combined abdominoperineal resection versus the segmental anterior resection. A 25-year postoperative follow-up in 301 patients. Am J Proctol. 1963;14:258-61.

7. Rothenberger DA, Wong WD. Abdominoperineal resection for adenocarcinoma of the low rectum. World J Surg. 1992;16:478-85.

8. Dehni N, McFadden N, McNamara DA, Guiguet M, Tiret E, Parc R. Oncologic results following abdominoperineal resection for adenocarcinoma of the low rectum. Dis Colon Rectum. 2003;46:867-74.

9. Kim JC, Yu CS, Lim SB, Kim CW, Kim JH, Kim TW. Abdominoperineal resection and low anterior resection: comparison of long-term oncologic outcome in matched patients with lower rectal cancer. Int J Colorectal Dis. 2013;28:493-501.

10. Roig-Vila JV, García-Armengol J, Bruna-Esteban M, Redondo-Cano C, Tornero-Ibáñez F, García-Aguadob R. Operating position in colorectal surgery. The importance of the basics. CIR ESP. 2009;86:204-12.

11. West NP, Anderin C, Smith KJ, Holm T, Quirke P. European Extralevator Abdominoperineal Excision Study G. Multicentre experience with extralevator abdominoperineal excision for low rectal cancer. Br J Surg. 2010;97:588-99.

12. Anderin C, Granath F, Martling A, Holm T. Local recurrence after prone versus supine abdomino-perineal excision for low rectal cancer. Colorectal Dis. 2013;15:812-5.

13. Showalter SL, Kelz RR, Mahmoud NN. Effect of technique on postoperative perineal wound infections in abdominoperineal resection. Am J Surg. 2013;206:80-5.

14. de Campos-Lobato LF, Stocchi L, Dietz DW, Lavery IC, Fazio WW, Kalady MF. Prone or lithotomy positioning during an abdominoperineal resection for rectal cancer results in comparable oncologic outcomes. Dis Colon Rectum. 2011;54:939-46.

15. Heald RJ. The 'Holy Plane' of rectal surgery. J R Soc Med. 1988;81:503-8.

16. Johar RS, Smith RP. Assessing gravimetric estimation of intraoperative blood loss. J Gynecol Surg. 1993;9:151-4.

17. Marr R, Birbeck K, Garvican J, Macklin CP, Tiffin N, Parsons WJ, et al. The modern abdominoperineal excision: the next challenge after total mesorectal excision. Ann Surg. 2005;242:74-82.

18. Chuwa EW, Seow-Choen F. Outcomes for abdominoperineal resections are not worse than those of anterior resections. Dis Colon Rectum. 2006;49:41-9.

19. Perry WB, Connaughton JC. Abdominoperineal resection: how is it done and what are the results? Clin Colon Rectal Surg. 2007;20:213-20.

20. Murrell ZA, Dixon MR, Vargas H, Arnell TD, Kumar R, Stamos MJ. Contemporary indications for and early outcomes of abdominoperineal resection. Am Surg. 2005;71:837-40.

21. Molina Rodriguez JL, Flor-Lorente B, Frasson M, Garcia-Botello S, Esclapez P, Espi $A$, et al. Low rectal cancer: abdominoperineal resection or low Hartmann resection? A postoperative outcome analysis. Dis Colon Rectum. 2011;54:958-62.

22. Pocard M, Zinzindohoue F, Haab F, Caplin S, Parc R, Tiret E. A prospective study of sexual and urinary function before and after total mesorectal excision with autonomic nerve preservation for rectal cancer. Surgery. 2002;131:368-72.

23. Bullard KM, Trudel JL, Baxter NN, Rothenberger DA. Primary perineal wound closure after preoperative radiotherapy and abdominoperineal resection has a high incidence of wound failure. Dis Colon Rectum. 2005;48:438-43.

24. de Haas WG, Miller MJ, Temple WJ, Kroll SS, Schusterman MA, Reece GP, et al. Perineal wound closure with the rectus abdominis musculocutaneous flap after tumor ablation. Ann Surg Oncol. 1995;2:400-6.

25. Chambers W, Khan A, Waters R, Lindsey I, George B, Mortensen N, et al. Examination of outcome following abdominoperineal resection for adenocarcinoma in Oxford. Colorectal Dis. 2010;12:1192-7.

26. Tekkis PP, Heriot AG, Smith J, Thompson MR, Finan P, Stamatakis JD. Comparison of circumferential margin involvement between restorative and nonrestorative resections for rectal cancer. Color Dis. 2005;7:369-74.

27. Williams NS, Johnston D. Survival and recurrence after sphincter saving resection and abdominoperineal resection for carcinoma of the middle third of the rectum. Br J Surg. 1984;71:278-82.

28. Williams NS, Durdey $P$, Johnston D. The outcome following sphincter-saving resection and abdominoperineal resection for low rectal cancer. Br J Surg. 1985;72:595-8

29. Adam IJ, Mohamdee MO, Martin IG, Scott N, Finan PJ, Johnston D, et al. Role of circumferential margin involvement in the local recurrence of rectal cancer. Lancet. 1994;344:707-11. 
30. Birbeck KF, Macklin CP, Tiffin NJ, Parsons W, Dixon MF, Mapstone NP, et al. Rates of circumferential resection margin involvement vary between surgeons and predict outcomes in rectal cancer surgery. Ann Surg. 2002;235:449-57.

31. Nagtegaal ID, Quirke P. What is the role for the circumferential margin in the modern treatment of rectal cancer? J Clin Oncol. 2008;26:303-12.

Submit your next manuscript to BioMed Central and take full advantage of:

- Convenient online submission

- Thorough peer review

- No space constraints or color figure charges

- Immediate publication on acceptance

- Inclusion in PubMed, CAS, Scopus and Google Scholar

- Research which is freely available for redistribution 